\title{
The Kirchhoff Index of Hypercubes and Related Complex Networks
}

\author{
Jiabao Liu, ${ }^{1,2,3}$ Jinde Cao, ${ }^{1,4}$ Xiang-Feng Pan, ${ }^{2}$ and Ahmed Elaiw ${ }^{4}$ \\ ${ }^{1}$ Department of Mathematics, Southeast University, Nanjing 210096, China \\ ${ }^{2}$ School of Mathematical Sciences, Anhui University, Hefei 230601, China \\ ${ }^{3}$ Anhui Xinhua University, Hefei 230088, China \\ ${ }^{4}$ Department of Mathematics, Faculty of Sciences, King Abdulaziz University, Jeddah, Saudi Arabia
}

Correspondence should be addressed to Jinde Cao; jdcao@seu.edu.cn

Received 14 October 2013; Accepted 31 October 2013

Academic Editor: Guanghui Wen

Copyright (C) 2013 Jiabao Liu et al. This is an open access article distributed under the Creative Commons Attribution License, which permits unrestricted use, distribution, and reproduction in any medium, provided the original work is properly cited.

\begin{abstract}
The resistance distance between any two vertices of $G$ is defined as the network effective resistance between them if each edge of $G$ is replaced by a unit resistor. The Kirchhoff index $\operatorname{Kf}(G)$ is the sum of resistance distances between all the pairs of vertices in $G$. We firstly provided an exact formula for the Kirchhoff index of the hypercubes networks $Q_{n}$ by utilizing spectral graph theory. Moreover, we obtained the relationship of Kirchhoff index between hypercubes networks $Q_{n}$ and its three variant networks $l\left(Q_{n}\right)$, $s\left(Q_{n}\right), t\left(Q_{n}\right)$ by deducing the characteristic polynomial of the Laplacian matrix related networks. Finally, the special formulae for the Kirchhoff indexes of $l\left(Q_{n}\right), s\left(Q_{n}\right)$, and $t\left(Q_{n}\right)$ were proposed, respectively.
\end{abstract}

\section{Introduction}

In this work we are concerned with finite undirected connected simple graphs. For the graph theoretical definitions and notations we follow [1]. A network is usually modelled by connected graphs $G=(V, E)$, where $V$ denotes the set of processors and $E$ denotes the set of communication links between processors. It is well known that the standard distance between two vertices of $G$, denoted by $d_{i j}$, is the shortest path connecting the two vertices. The Wiener index [2], denoted by $W(G)$, is a famous distance-based topological index and is defined as the sum of distances between all the pairs of vertices in $G$ :

$$
W(G)=\sum_{i<j} d_{i j}(G) .
$$

As an analogue to the Wiener index $W(G)$, another novel distance function named resistance distance was firstly proposed by Klein and Randić [3]. The resistance distance between two arbitrary in an electrical networks, many properties over resistance distances have been actually proved $[2,4-9]$. The resistance distance between any two vertices of $G$ is defined as the networks effective resistance between them if each edge of $G$ is replaced by a unit resistor. They also defined the Kirchhoff index $\operatorname{Kf}(G)$ of $G$ as the sum of resistance distances between all pairs of vertices in $G$; that is,

$$
\mathrm{Kf}(G)=\sum_{i<j} r_{i j}(G) .
$$

Klein and Randić [3] proved that $r_{i j} \leq d_{i j}$ and $\mathrm{Kf}(G) \leq$ $W(G)$ with equality if and only if $G$ is a tree; it is shown that the Kirchhoff index has very nice purely mathematical and physical interpretations.

The Kirchhoff index has wide applications in physical interpretations, electric circuit, graph theory, and chemistry [10-15]. For example, Zhu et al. [16] and Gutman and Mohar [17] proved that the Kirchhoff index of a graph or networks is the sum of reciprocal nonzero Laplacian eigenvalues of the graph or networks multiplied by the number of the vertices. The Kirchhoff index also is a structure descriptor like the Wiener index [9]. The Kirchhoff index has been computed for cycles [4], geodetic graphs [5], and some fullerenes including buckminsterfullerenes [6]. The Kirchhoff index of some product graphs, join graphs, and corona graphs was 
studied [8]. More results of the applications on Kirchhoff index were explored in $[2,7,10,14]$.

The hypercubes network $Q_{n}$ obtained considerable attention due to its perfect properties, such as symmetry, regular structure, strong connectivity, and small diameter $[18,19]$. For more results on the hypercubes network and its applications, see [18-25]. As the importance the hypercubes networks $Q_{n}$, many variants of it were presented, among which, for instance, are generalized hypercubes, folded hypercubes, the line graphs of hypercubes $l\left(Q_{n}\right)$, the subdivision graphs of hypercubes $s\left(Q_{n}\right)$, and the total graphs of hypercubes $t\left(Q_{n}\right)$ $[19,20]$.

The hypercubes networks $Q_{n}$ may be constructed from the family of subsets of a set with a binary string of length $n$, by making a vertex for each possible subset and joining two vertices by an edge whenever the corresponding subsets differ in a single binary string. The hypercubes networks $Q_{n}$ admits several definitions of which two are stated as follows [26].

Definition 1 (see [26]). The hypercubes networks $Q_{n}$ has $2^{n}$ vertices each labelled with a binary string of length $n$. Two vertices $X=x_{1} x_{2} \cdots x_{n}$ and $Y=y_{1} y_{2} \cdots y_{n}$ are adjacent if and only if there exists an $i, 1 \leq i \leq n$, such that $x_{i}=\overline{y_{i}}$, where $\overline{y_{i}}$ denoted the complement of binary digit $y_{i}$ and $x_{j}=y_{j}$, for all $j \neq i$, and $1 \leq j \leq n$.

Definition 2 (recursive construction [26]). The hypercubes network $Q_{n}$ is recursively constructed by taking two copies of $Q_{n-1}$, denoted by $Q_{n-1}^{0}$ and $Q_{n-1}^{1}$, and adding $2^{n-1}$ edges as follows: let $V\left(Q_{n-1}^{0}\right)=\left\{0 U=0 u_{2} u_{3} \cdots u_{n}: u_{i}=0\right.$ or 1$\}$ and $V\left(Q_{n-1}^{1}\right)=\left\{1 V=1 v_{2} v_{3} \cdots v_{n}: v_{i}=0\right.$ or 1$\}$. A vertex $0 U=$ $0 u_{2} u_{3} \cdots u_{n}$ of $Q_{n-1}^{0}$ is joined to a vertex $1 V=1 v_{2} v_{3} \cdots v_{n}$ of $Q_{n-1}^{1}$ if and only if $u_{i}=v_{i}$ for every $i, 2 \leq i \leq n$.

At the end of [10], the authors presented a problem to consider the Kirchhoff index derived from a single graph, such as the line graph, the subdivision graph and the total graph Gao et al. [27] obtained special formulae for the Kirchhoff index of $l(G), s(G)$, and $t(G)$, where $G$ is a regular graph. Motivated by the previous results, we present the corresponding formulae for the Kirchhoff index of the hypercubes network $Q_{n}$ and its three variant networks $l\left(Q_{n}\right), s\left(Q_{n}\right)$, and $t\left(Q_{n}\right)$ in this paper.

The remainder of this paper is organized as follows. Section 2 gives some basic notations and some preliminaries in our discussion. The proofs of our main results are in Section 3. Finally, some conclusions are given in Section 4 .

\section{Notations and Some Preliminaries}

In this section, we recall some basic notations and results in graphs theory. The adjacency matrix $A(G)$ of $G$ is an $n \times n$ matrix with the $(i, j)$-entry equal to 1 if vertices $i$ and $j$ are adjacent and 0 otherwise. Suppose that $D(G)=$ $\operatorname{diag}\left(d_{1}(G), d_{2}(G), \ldots, d_{n}(G)\right)$ is the degree diagonal matrix of $G$, where $d_{i}(G)$ is the degree of the vertex $i, i=1,2, \ldots, n$. Let $L(G)=D(G)-A(G)$ be called the Laplacian matrix of $G$. Then the eigenvalues of $A(G)$ and $L(G)$ are called eigenvalues and
Laplacian eigenvalues of $G$, respectively. For more details the readers may refer to [1].

Yin and Wang [28] proved the following Lemma.

Lemma 3 (see [28]). For the hypercubes networks $Q_{n}$ with $n \geq$ 2 ,

$$
\operatorname{Spec}\left(Q_{n}\right)=\left(\begin{array}{cccccc}
0 & 2 & \cdots & 2 i & \cdots & 2 n \\
C_{n}^{0} & C_{n}^{1} & \cdots & C_{n}^{i} & \cdots & C_{n}^{n}
\end{array}\right),
$$

where the $2 i(i=0,1, \ldots, n)$ are the eigenvalues of the Laplacian matrix of hypercubes networks, and $C_{n}^{i}$ are the multiplicities of the eigenvalues $2 i$.

Gutman and Mohar [17] and Zhu et al. [16] presented the Kirchhoff index of a graph in terms of Laplacian eigenvalues as follows.

Lemma 4 (see $[16,17])$. Let $G$ be a connected graph with $n \geq 2$ vertices; then

$$
\operatorname{Kf}(G)=n \sum_{i=1}^{n-1} \frac{1}{\lambda_{i}} .
$$

Let $P_{(G)}(x)$ be the characteristic polynomial of the Laplacian matrix of a graph $G$, the following results were shown in [27].

Lemma 5 (see [27]). Let $G$ be an r-regular connected graph with $n$ vertices and $m$ edges; then

$$
\begin{gathered}
P_{l(G)}(x)=(x-2 r)^{m-n} P_{G}(x), \\
P_{s(G)}(x)=(-1)^{m}(2-x)^{m-n} P_{G}(x(r+2-x)), \\
P_{t(G)}(x)=(-1)^{m}(r+1-x)^{n} \\
\quad \times(2 r+2-x)^{m-n} P_{G}\left(\frac{x(r+2-x)}{r-x+1}\right),
\end{gathered}
$$

where $P_{l(G)}(x), P_{s(G)}(x)$, and $P_{t(G)}(x)$ are the characteristic polynomials for the Laplacian matrix of graphs $l(G), s(G)$, and $t(G)$, respectively.

It is worthwhile to note that the conclusion of Lemma 5 is not completely correct, the authors [29] recently show the Laplacian characteristic polynomial of $t(G)$, where $G$ is a regular graph, which correct the Lemma 5 in Gao et al. [27] (2012) as follows.

Lemma 6 (see [29]). Let G be a r-regular connected graph with $n$ vertices and $m$ edges, then

$$
\begin{aligned}
P_{s(G)}(x) & =(-1)^{n}(2-x)^{m-n} P_{G}(x(r+2-x)), \\
P_{t(G)}(x)=x & (x-r-2)(x-2 r-2)^{m-n} \\
\times & \prod_{i=1}^{n-1}\left[\left(x^{2}-2 x-r x\right)+(3-2 x+r) \mu_{i}+\mu_{i}^{2}\right] .
\end{aligned}
$$

where $P_{s(G)}(x), P_{t(G)}(x)$ are the characteristic polynomial for the Laplacian matrix of graphs $s(G)$ and $t(G)$, respectively. 
The following Lemma give an expression on $\operatorname{Kf}(t(G))$ and $\mathrm{Kf}(G)$ of a regular graph $G$.

Lemma 7 (see [29]). Let G be a r-regular connected graph with $n$ vertices and $m$ edges, and $r \geq 2$, then

$$
\begin{array}{r}
\mathrm{Kf}(t(G))=\frac{(r+2)^{2}}{2(r+3)} \operatorname{Kf}(G)+\frac{n^{2}\left(r^{2}-4\right)}{8(r+1)}+\frac{n}{2} \\
+\frac{n(r+2)(r+4)}{2(r+3)} \sum_{i=1}^{n-1} \frac{1}{\mu_{i}+3+r} .
\end{array}
$$

For proving the formula of the Kirchhoff index on the subdivision graph of hypercubes, we prove the following Lemma by utilizing Vieta's Theorem; in our proof, some techniques in [27] are referred to.

Lemma 8. Let $P_{\mathrm{Q}_{n}}(x)$ be the characteristic polynomial of the Laplacian matrix of the hypercubes networks $Q_{n}$ with $n \geq 2$ and

$$
P_{\mathrm{Q}_{n}}(x)=x^{2^{n}}+a_{1} x^{2^{n}-1}+a_{2} x^{2^{n}-2}+\cdots+a_{2^{n}-1} x ;
$$

then

$$
\frac{\operatorname{Kf}\left(Q_{n}\right)}{2^{n}}=-\frac{a_{2^{n}-2}}{a_{2^{n}-1}},
$$

where $a_{2^{n}-1}, a_{2^{n}-2}$ are the coefficients of $x$ and $x^{2}$ in the characteristic polynomial, respectively.

Proof. Let $\operatorname{Spec}\left(Q_{n}\right)=\left(\lambda_{0}, \lambda_{1}, \lambda_{2}, \ldots \lambda_{n}, \lambda_{n+1}, \ldots, \lambda_{2^{n}-1}\right)$. Then $\lambda_{i}, i=1,2, \ldots, 2^{n}-1$ satisfy the following equation:

$$
x^{2^{n}-1}+a_{1} x^{2^{n}-2}+\cdots+a_{2^{n}-1}=0 ;
$$

it is not difficult to check that $1 / \lambda_{i}, i=1,2, \ldots, 2^{n}-1$ are the roots of equation

$$
a_{2^{n}-1} x^{2^{n}-1}+a_{2^{n}-2} x^{2^{n}-2}+\cdots+a_{1} x+1=0 .
$$

Note that $Q_{n}$ is connected graph and the multiplicity of 0 as an eigenvalue of $L\left(Q_{n}\right)$ is equal to the number of the connected components in $Q_{n}$. So, $a_{2^{n}-1} \neq 0$; by Lemma 4 and Vieta's Theorem

$$
\frac{\mathrm{Kf}\left(Q_{n}\right)}{2^{n}}=\sum_{i=1}^{2^{n}-1} \frac{1}{\lambda_{i}}=-\frac{a_{2^{n}-2}}{a_{2^{n}-1}},
$$

where $a_{2^{n}-1}, a_{2^{n}-2}$ are the coefficients of $x$ and $x^{2}$ in the characteristic polynomial of the Laplacian matrix of the hypercubes networks $Q_{n}$.

\section{Main Results}

3.1. The Kirchhoff Index in Hypercubes Networks $Q_{n}$. In this section, we firstly give formula for the Kirchhoff index in the hypercubes $Q_{n}$ with $n \geq 2$.
Theorem 9. For the hypercubes networks $Q_{n}$ with $n \geq 2$,

$$
\mathrm{Kf}\left(Q_{n}\right)=2^{n} \sum_{i=1}^{n} \frac{C_{n}^{i}}{2 i},
$$

where the $2 i(i=1, \ldots, n)$ are the eigenvalues of the Laplacian matrix of hypercubes networks and the binomial coefficients $C_{n}^{i}$ are the multiplicities of the eigenvalues $2 i$.

Proof. Since the hypercubes networks $Q_{n}$ have $2^{n}$ vertices and $n 2^{n-1}$ edges, then by Lemma 3 ,

$$
\operatorname{Spec}\left(Q_{n}\right)=\left(\begin{array}{cccccc}
0 & 2 & \cdots & 2 i & \cdots & 2 n \\
C_{n}^{0} & C_{n}^{1} & \cdots & C_{n}^{i} & \cdots & C_{n}^{n}
\end{array}\right)
$$

then

$$
\begin{aligned}
\operatorname{Kf}\left(Q_{n}\right)= & 2^{n} \sum_{i=1}^{2^{n}-1} \frac{1}{\lambda_{i}} \\
= & 2^{n}\left(\frac{C_{n}^{1}}{2 \times 1}+\frac{C_{n}^{2}}{2 \times 2}+\cdots\right. \\
& \left.\quad+\frac{C_{n}^{i}}{2 \times n-1}+\cdots+\frac{C_{n}^{n}}{2 \times n}\right) \\
= & 2^{n} \sum_{i=1}^{n} \frac{C_{n}^{i}}{2 i} .
\end{aligned}
$$

The proof of Theorem 9 is completed.

Remark 10. Palacios and Renom studied the Kirchhoff index of the d-dimensional hypercube in [5] by using probabilistic tools, where they obtained a closed-form formula for the Kirchhoff index and found the asymptotic value $2^{2 d} / d$. We present the formula for Kirchhoff index by directly calculating the eigenvalues of the Laplacian matrix of hypercubes networks, which is different from their technique and results.

3.2. The Kirchhoff Index in the Line Graph of Hypercubes Networks $l\left(Q_{n}\right)$. The line graph of a graph $G$, denoted by $l(G)$, is the graph whose vertices correspond to the edges of $G$, in which two vertices are adjacent if and only if the corresponding edges of $G$ share a common vertex. In the following theorem, we proposed a formula for the Kirchhoff index in the line graph of hypercubes networks $l\left(Q_{n}\right)$, denoted by $\operatorname{Kf}\left(l\left(Q_{n}\right)\right)$, where the eigenvalues of $Q_{n}$ are $\lambda_{0} \leq \lambda_{1} \leq \lambda_{2} \leq$ $\cdots \leq \lambda_{n} \leq \lambda_{n+1} \leq \cdots \leq \lambda_{2^{n}-1}$.

Theorem 11. Let $l\left(Q_{n}\right)$ be line graphs of hypercubes $Q_{n}$ with $n \geq 2$; then

$$
\operatorname{Kf}\left(l\left(Q_{n}\right)\right)=n 2^{n-1} \sum_{i=1}^{n} \frac{C_{n}^{i}}{2 i}++n 2^{2 n-3}-2^{2 n-2},
$$

where the $2 i(i=1, \ldots, n)$ are the eigenvalues of the Laplacian matrix of hypercubes networks and the binomial coefficients $C_{n}^{i}$ are the multiplicities of the eigenvalues $2 i$. 
Proof. Now, for convenience, we denoted the numbers of vertices and edges in the hypercubes networks $Q_{n}$ by $p$ and $q$, respectively. Obviously, $p=2^{n}, q=n 2^{n-1}$, so the line graphs of hypercubes $l\left(Q_{n}\right)$ have $q=n 2^{n-1}$ vertices.

By Lemma 5,

$$
P_{l\left(Q_{n}\right)}(x)=(x-2 r)^{q-p} P_{G}(x) .
$$

Comparing the spectrum of $Q_{n}$,

$$
\operatorname{Spec}\left(Q_{n}\right)=\left(\begin{array}{cccccc}
0 & 2 & \cdots & 2 i & \cdots & 2 n \\
C_{n}^{0} & C_{n}^{1} & \cdots & C_{n}^{i} & \cdots & C_{n}^{n}
\end{array}\right) .
$$

We can easily obtain the spectrum of $l\left(Q_{n}\right)$ as follows:

$$
\operatorname{Spec}\left(l\left(Q_{n}\right)\right)=\left(\begin{array}{lllllll}
2 n & 2 n & \cdots & 2 n & \lambda_{0} & \cdots & \lambda_{2_{n}-1}
\end{array}\right)
$$

where $\lambda_{0}, \lambda_{1}, \ldots \lambda_{n}, \lambda_{n+1}, \ldots, \lambda_{2^{n}-1}$ are the eigenvalues of $Q_{n}$ and $\lambda_{0} \leq \lambda_{1} \leq \lambda_{2} \leq \cdots \lambda_{n} \leq \lambda_{n+1} \leq \cdots \leq \lambda_{2^{n}-1}$.

Notice that the line graphs of hypercubes $l\left(Q_{n}\right)$ have $q=$ $n 2^{n-1}$ vertices; by Lemma 4 ,

$$
\begin{aligned}
\mathrm{Kf}\left(l\left(Q_{n}\right)\right) & =q\left(\sum_{i=1}^{2^{n}-1} \frac{1}{\lambda_{i}}+\frac{q-p}{2 n}\right) \\
& =\frac{n 2^{n-1}}{2^{n}} \mathrm{Kf}\left(Q_{n}\right)+\frac{q^{2}-p q}{2 n} \\
& =\frac{n}{2} \operatorname{Kf}\left(Q_{n}\right)+n 2^{2 n-3}-2^{2 n-2} .
\end{aligned}
$$

Substituting the results of Theorem 9 (15) into (22), we can get the formula for the Kirchhoff index on the line graph of hypercubes $\operatorname{Kf}\left(l\left(Q_{n}\right)\right)$ as follows:

$$
\operatorname{Kf}\left(l\left(Q_{n}\right)\right)=n 2^{n-1} \sum_{i=1}^{n} \frac{C_{n}^{i}}{2 i}+n 2^{2 \mathrm{n}-3}-2^{2 n-2} .
$$

This completes the proof.

3.3. The Kirchhoff Index in the Subdivision Graph of Hypercubes Networks $s\left(Q_{n}\right)$. The subdivision graph of a graph $G$, denoted by $s(G)$, is the graph obtained by replacing every edge in $G$ with a copy of $P_{2}$ (path of length two). In an almost identical way as Theorem 11, we required the formula for the Kirchhoff index in the subdivision graph of hypercubes $s\left(Q_{n}\right)$, denoted by $\operatorname{Kf}\left(s\left(Q_{n}\right)\right)$.

Theorem 12. Let $s\left(Q_{n}\right)$ be subdivision graphs of the hypercubes $Q_{n}$ with $n \geq 2$; then

$$
\begin{aligned}
\operatorname{Kf}\left(s\left(Q_{n}\right)\right)= & (n+2)\left(2^{n}+n 2^{n-1}\right) \\
& \times \sum_{i=1}^{n} \frac{C_{n}^{i}}{2 i}+2^{n-1}+n^{2} 2^{2 n-3}-2^{2 n-1},
\end{aligned}
$$

where the $2 i(i=1, \ldots, n)$ are the eigenvalues of the Laplacian matrix of hypercubes networks and the binomial coefficients $C_{n}^{i}$ are the multiplicities of the eigenvalues $2 i$.
Proof. Now supposing that $n \geq 2, P_{\left(Q_{n}\right)}(x)$ is the characteristic polynomial of the Laplacian matrix of the hypercubes $Q_{n}$, $P_{\mathrm{Q}_{n}}(x)=x^{2^{n}}+a_{1} x^{2^{n}-1}+a_{2} x^{2^{n}-2}+\cdots+a_{2^{n}-1} x$, and

$$
\operatorname{Spec}\left(Q_{n}\right)=\left\{\lambda_{0}, \lambda_{1}, \lambda_{2}, \ldots \lambda_{n}, \lambda_{n+1}, \ldots, \lambda_{2^{n}-1}\right\},
$$

where $\lambda_{i}, i=0,1,2, \ldots, 2^{n}-1$ are the Laplacian eigenvalues of $Q_{n}$ Then by Lemma 8,

$$
\frac{\mathrm{Kf}\left(Q_{n}\right)}{2^{n}}=-\frac{a_{2^{n}-2}}{a_{2^{n}-1}} .
$$

By Lemma 5, we have

$$
\begin{aligned}
P_{s\left(Q_{n}\right)}(x)=(-1)^{p}(2-x)^{q-p} \\
\times\left[x^{2^{n}}(n+2-x)^{2^{n}}+a_{1} x^{2^{n}-1}(n+2-x)^{2^{n}-1}\right. \\
\quad+\cdots+a_{2^{n}-2} x^{2}(n+2-x)^{2} \\
\left.\quad+a_{2^{n}-1} x(n+2-x)\right] .
\end{aligned}
$$

Consequently, the coefficient of $x^{2}$ in $P_{s\left(\mathrm{Q}_{n}\right)}(x)$ is

$$
\begin{aligned}
a_{2^{n}-2}=(-1)^{p} 2^{q-p}[ & (n+2)^{2} a_{2^{n}-2}-2^{q-p} a_{2^{n}-1} \\
& \left.-(q-p) 2^{q-p-1}(n+2) a_{2^{n}-1}\right],
\end{aligned}
$$

and the coefficient of $x$ in $P_{s\left(Q_{n}\right)}(x)$ is

$$
(-1)^{p} 2^{q-p}(n+2) a_{2^{n}-1}
$$

Note that $s\left(Q_{n}\right)$ has $2^{n}+n 2^{n-1}$ vertices. By Lemma 6 and substituting the coefficients into (26),

$$
\begin{aligned}
\frac{\mathrm{Kf}(s(G))}{2^{n}+n 2^{n-1}} & \\
= & -\left(2^{q-p}(n+2)^{2} a_{2^{n}-2}-2^{q-p} a_{2^{n}-1}\right. \\
& \left.\quad-(q-p) 2^{q-p-1} a_{2^{n}-1}(n+2)\right) \\
& \times\left(2^{q-p} a_{2^{n}-1}(n+2)\right)^{-1} \\
= & -\frac{a_{2^{n}-2}(n+2)}{a_{2^{n}-1}}+\frac{1}{n+2}+\frac{q-p}{2} .
\end{aligned}
$$

By substituting $q=n 2^{n-1}, p=2^{n}$ and the results of Lemma 5 into (31), we have

$$
\begin{aligned}
& \frac{\operatorname{Kf}\left(s\left(Q_{n}\right)\right)}{2^{n}+n 2^{n-1}} \\
& =-\frac{a_{2^{n}-2}(n+2)}{a_{2^{n}-1}}+\frac{1}{n+2}+\frac{n 2^{n-1}-2^{n}}{2} \\
& =\frac{(n+2) \operatorname{Kf}\left(Q_{n}\right)}{2^{n}}+\frac{1}{n+2}+\frac{n 2^{n-1}-2^{n}}{2} .
\end{aligned}
$$


Simplifying (33) and substituting the results of Theorem 9 (15), we can get the formula of the Kirchhoff index on the subdivision graph of the hypercubes $\operatorname{Kf}\left(s\left(Q_{n}\right)\right)$ as follows:

$$
\begin{aligned}
\mathrm{Kf}\left(s\left(Q_{n}\right)\right)= & \frac{(n+2)\left(2^{n}+n 2^{n-1}\right)}{2^{n}} \mathrm{Kf}\left(Q_{n}\right) \\
& +\frac{2^{n}+n 2^{n-1}}{n+2}+\frac{\left(2^{n}+n 2^{n-1}\right)\left(n 2^{n-1}-2^{n}\right)}{2} \\
= & \frac{(n+2)\left(2^{n}+n 2^{n-1}\right)}{2^{n}} \mathrm{Kf}\left(Q_{n}\right) \\
& +2^{n-1}+n^{2} 2^{2 n-3}-2^{2 n-1} \\
= & (n+2)\left(2^{n}+n 2^{n-1}\right) \\
& \times \sum_{i=1}^{n} \frac{C_{n}^{i}}{2 i}+2^{n-1}+n^{2} 2^{2 n-3}-2^{2 n-1} .
\end{aligned}
$$

Thus, the results of the Theorem 12 hold.

3.4. The Kirchhoff Index in the Total Graph of Hypercubes Networks $t\left(Q_{n}\right)$. The total graph of a graph $G$, denoted by $t(G)$, is the graph whose vertices correspond to the union of the set of vertices and edges of $G$, with two vertices of $t(G)$ being adjacent if and only if the corresponding elements are adjacent or incident in $G$. We now proved the formula of the Kirchhoff index in the total graph of the hypercubes networks $t\left(Q_{n}\right)$, denoted by $\operatorname{Kf}\left(t\left(Q_{n}\right)\right)$.

Theorem 13. Let $t\left(Q_{n}\right)$ be the total graphs of the hypercubes networks $Q_{n}$ with $n \geq 2$; then

$$
\begin{aligned}
\operatorname{Kf}\left(t\left(Q_{n}\right)\right) & \\
= & \frac{2^{n-1}(n+2)^{2}}{n+3} \sum_{i=1}^{n} \frac{C_{n}^{i}}{2 i}+\frac{n^{4}-4 n^{2}}{8(n+1)} \\
& +\frac{n}{2}+\frac{\left(n^{2}+2 n\right)(n+4)}{2(n+3)} \sum_{i=1}^{n} \frac{C_{n}^{i}}{2 i+3+n},
\end{aligned}
$$

where the $2 i(i=0,1, \ldots, n)$ are the eigenvalues of the Laplacian matrix of hypercubes networks and the binomial coefficients $C_{n}^{i}$ are the multiplicities of the eigenvalues $2 i$.

Proof. Let

$$
P_{t\left(\mathrm{Q}_{n}\right)}(x)=x^{2^{n}}+a_{1} x^{2^{n}-1}+a_{2} x^{2^{n}-2}+\ldots+a_{2^{n}-1} x .
$$

Then by Lemma 8 ,

$$
\frac{\operatorname{Kf}\left(t\left(Q_{n}\right)\right)}{2^{n}}=-\frac{a_{2^{n}-2}}{a_{2^{n}-1}} .
$$

Applying Lemma 6, the Laplacian characteristic polynomial of $t\left(Q_{n}\right)$ is

$$
\begin{aligned}
P_{t\left(\mathrm{Q}_{n}\right)}(x)= & x(x-n-2)(x-2 n-2)^{q-p} \\
& \times \prod_{i=1}^{2^{n}-1}\left\{\left[x^{2}-2 x-n x\right]+[3-2 x+n] \lambda_{i}+\lambda_{i}^{2}\right\} \\
= & x(x-n-2)(x-2 n-2)^{q-p} \\
& \times \prod_{i=1}^{2^{n}-1}\left[x^{2}-\left(2+n+2 \lambda_{i}\right) x+\lambda_{i}^{2}+(n+3) \lambda_{i}\right] .
\end{aligned}
$$

Consider that $Q_{n}$ has $2^{n}$ vertices and $r=n$, by Lemma 7 , we can get the following equality,

$$
\begin{array}{r}
\mathrm{Kf}\left(t\left(Q_{n}\right)\right)=\frac{(r+2)^{2}}{2(r+3)} \operatorname{Kf}\left(Q_{n}\right)+\frac{n^{2}\left(r^{2}-4\right)}{8(r+1)}+\frac{n}{2} \\
+\frac{n(r+2)(r+4)^{2^{n}-1}}{2(r+3)} \sum_{i=1}^{1} \frac{1}{\lambda_{i}+3+r} \\
=\frac{(n+2)^{2}}{2(n+3)} \operatorname{Kf}\left(Q_{n}\right)+\frac{n^{4}-4 n^{2}}{8(n+1)}+\frac{n}{2} \\
+\frac{\left(n^{2}+2 n\right)(n+4)^{2^{n}-1}}{2(n+3)} \sum_{i=1}^{\frac{1}{\lambda_{i}+3+n}} .
\end{array}
$$

Consequently, the relationships between the hypercubes networks $Q_{n}$ and its variant networks $t\left(Q_{n}\right)$ for Kirchhoff index is

$$
\begin{aligned}
\operatorname{Kf}\left(t\left(Q_{n}\right)\right)= & \frac{(n+2)^{2}}{2(n+3)} \operatorname{Kf}\left(Q_{n}\right)+\frac{n^{4}-4 n^{2}}{8(n+1)}+\frac{n}{2} \\
& +\frac{\left(n^{2}+2 n\right)(n+4)}{2(n+3)} \sum_{i=1}^{2^{n}-1} \frac{1}{\lambda_{i}+3+n} .
\end{aligned}
$$

Substitutes the result of Theorem 9 and simplifies about equation, we can get the formula for the Kirchhoff index on the total graph of hypercubes networks $\operatorname{Kf}\left(t\left(Q_{n}\right)\right)$ as follows.

$$
\begin{aligned}
\operatorname{Kf}\left(t\left(Q_{n}\right)\right)= & \frac{(n+2)^{2}}{2(n+3)} \operatorname{Kf}\left(Q_{n}\right)+\frac{n^{4}-4 n^{2}}{8(n+1)}+\frac{n}{2} \\
& +\frac{\left(n^{2}+2 n\right)(n+4)}{2(n+3)} \sum_{i=1}^{2^{n}-1} \frac{1}{\lambda_{i}+3+n} \\
= & \frac{2^{n-1}(n+2)^{2}}{n+3} \sum_{i=1}^{n} \frac{C_{n}^{i}}{2 i}+\frac{n^{4}-4 n^{2}}{8(n+1)}+\frac{n}{2} \\
& +\frac{\left(n^{2}+2 n\right)(n+4)}{2(n+3)} \sum_{i=1}^{n} \frac{C_{n}^{i}}{2 i+3+n} .
\end{aligned}
$$

This completes the proof of the Theorem. 


\section{Conclusions}

In this paper, we focused on the Kirchhoff index of the hypercubes networks and related networks, which are important networks topology indexes for parallel processing computer systems. We obtained some exact formulae for the Kirchhoff index of the hypercubes networks $Q_{n}$ and related networks by utilizing spectral graph theory, such as $\operatorname{Kf}\left(Q_{n}\right)=$ $2^{n} \sum_{i=1}^{n}\left(C_{n}^{i} / 2 i\right)$, where the $2 i(i=1, \ldots, n)$ are the eigenvalues of the Laplacian matrix of hypercubes networks and the binomial coefficients $C_{n}^{i}$ are the multiplicities of the eigenvalues $2 i$.

We also obtained the relationship for Kirchhoff index between hypercubes networks $Q_{n}$ and its three variant networks $l\left(Q_{n}\right), s\left(Q_{n}\right)$, and $t\left(Q_{n}\right)$, respectively, by deducing the characteristic polynomial of the Laplacian matrix related networks.

Finally, the special formulae for the Kirchhoff indexes of $l\left(Q_{n}\right), s\left(Q_{n}\right)$, and $t\left(Q_{n}\right)$ were proposed, respectively, by making use of spectral graph theory and Vieta's Theorem.

\section{Acknowledgments}

The work of Jinde Cao and Ahmed Elaiw was funded by the Deanship of Scientific Research (DSR), King Abdulaziz University (KAU), under Grant 3-130/1434/HiCi. The authors, therefore, acknowledge technical and financial support of KAU; the work of Jiabao Liu was supported by the Natural Science Foundation of Anhui Province of China under Grant no. KJ2013B015; the work of Xiangfeng Pan was supported by the National Science Foundation of China under Grant nos. 10901001, 11171097, and 11371028.

\section{References}

[1] J. M. Xu, Topological Strucure and Analysis of Interconnction Networks, Kluwer Academic Publishers, Dordrecht, The Netherlands, 2001.

[2] E. Estrada and N. Hatano, "Topological atomic displacements, Kirchhoff and Wiener indices of molecules," Chemical Physics Letters, vol. 486, no. 4-6, pp. 166-170, 2010.

[3] D. J. Klein and M. Randić, "Resistance distance," Journal of Mathematical Chemistry, vol. 12, no. 1-4, pp. 81-95, 1993.

[4] I. Lukovits, S. Nikolić, and N. Trinajstić, "Resistance distance in regular graphs," International Journal of Quantum Chemistry, vol. 71, no. 3, pp. 217-225, 1999.

[5] J. L. Palacios and J. M. Renom, "Bounds for the Kirchhoff index of regular graphs via the spectra of their random walks," International Journal of Quantum Chemistry, vol. 110, no. 9, pp. 1637-1641, 2010.

[6] P. W. Fowler, "Resistance distances in fullerene graphs," Croatica Chemica Acta, vol. 75, no. 2, pp. 401-408, 2002.

[7] H. Zhang, X. Jiang, and Y. Yang, "Bicyclic graphs with extremal Kirchhoff index," Communications in Mathematical and in Computer Chemistry, vol. 61, no. 3, pp. 697-712, 2009.

[8] C. Arauz, "The Kirchhoff indexes of some composite networks," Discrete Applied Mathematics, vol. 160, no. 10-11, pp. 1429-1440, 2012.

[9] W. Xiao and I. Gutman, "Resistance distance and Laplacian spectrum," Theoretical Chemistry Accounts, vol. 110, no. 4, pp. 284-289, 2003.
[10] H. Zhang, Y. Yang, and C. Li, "Kirchhoff index of composite graphs," Discrete Applied Mathematics, vol. 157, no. 13, pp. 29182927, 2009.

[11] M. Bianchi, A. Cornaro, J. L. Palacios, and A. Torriero, "Bounds for the Kirchhoff index via majorization techniques," Journal of Mathematical Chemistry, vol. 51, no. 2, pp. 569-587, 2013.

[12] J. L. Palacios and J. M. Renom, "Another look at the degreeKirchhoff index," International Journal of Quantum Chemistry, vol. 111, no. 14, pp. 3453-3455, 2011.

[13] H. Wang, H. Hua, and D. Wang, "Cacti with minimum, secondminimum, and third-minimum Kirchhoff indices," Mathematical Communications, vol. 15, no. 2, pp. 347-358, 2010.

[14] B. Zhou and N. Trinajstić, "Mathematical properties of molecular descriptors based on distances," Croatica Chemica Acta, vol. 83, no. 2, pp. 227-242, 2010.

[15] A. D. Maden, A. S. Cevik, I. N. Cangul, and K. C. Das, "On the Kirchhoff matrix, a new Kirchhoff index and the Kirchhoff energy," Journal of Inequalities and Applications, vol. 2013, article 337, 14 pages, 2013.

[16] H.-Y.Zhu, D. J. Klein, and I. Lukovits, "Extensions of the Wiener number," Journal of Chemical Information and Computer Sciences, vol. 36, no. 3, pp. 420-428, 1996.

[17] I. Gutman and B. Mohar, "The quasi-Wiener and the Kirchhoff indices coincide," Journal of Chemical Information and Computer Sciences, vol. 36, no. 5, pp. 982-985, 1996.

[18] J. Fink, "Perfect matchings extend to Hamilton cycles in hypercubes," Journal of Combinatorial Theory. Series B, vol. 97, no. 6, pp. 1074-1076, 2007.

[19] A. Bossard and K. Kaneko, "k-pairwise disjoint paths routing in perfect hierarchical hypercubes," The Journal of Supercomputing, vol. 14, no. 3, pp. 1-11, 2013.

[20] M. Liu and H. Liu, "Paths and cycles embedding on faulty enhanced hypercube networks," Acta Mathematica Scientia. Series B, vol. 33, no. 1, pp. 227-246, 2013.

[21] J.-H. Park, H.-S. Lim, and H.-C. Kim, "Panconnectivity and pancyclicity of hypercube-like interconnection networks with faulty elements," Theoretical Computer Science, vol. 377, no. 1-3, pp. 170-180, 2007.

[22] D. Wang and M. Lu, "Edge fault tolerance of super edge connectivity for three families of interconnection networks," Information Sciences, vol. 188, pp. 260-268, 2012.

[23] J.-M. Xu, J.-W. Wang, and W.-W. Wang, "On super and restricted connectivity of some interconnection networks," Ars Combinatoria, vol. 94, pp. 25-32, 2010.

[24] X.-J. Li and J.-M. Xu, "Edge-fault tolerance of hypercube-like networks," Information Processing Letters, vol. 113, no. 19-21, pp. 760-763, 2013.

[25] G. Wen, Z. Duan, W. Yu, and G. Chen, "Consensus in multiagent systems with communication constraints," International Journal of Robust and Nonlinear Control, vol. 22, no. 2, pp. 170$182,2012$.

[26] R. Indhumathi and S. A. Choudum, "Embedding certain height-balanced trees and complete $p^{m}$-ary trees into hypercubes," Journal of Discrete Algorithms, vol. 22, no. 1, pp. 53-65, 2013.

[27] X. Gao, Y. Luo, and W. Liu, "Kirchhoff index in line, subdivision and total graphs of a regular graph," Discrete Applied Mathematics, vol. 160, no. 4-5, pp. 560-565, 2012.

[28] J. H. Yin and R. G. Wang, "Spectra of Laplacian matrices of hypercubes," Journal of Zhejiang University. Science Edition, vol. 34, no. 3, pp. 321-323, 2007. 
[29] Z. You, L. You, and W. Hong, "Comment on Kirchhoff index in line, subdivision and total graphs of a regular graph," Discrete Applied Mathematics, vol. 161, no. 1, pp. 3100-3103, 2013. 


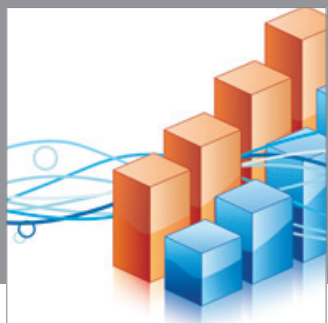

Advances in

Operations Research

mansans

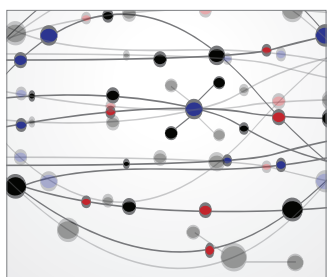

The Scientific World Journal
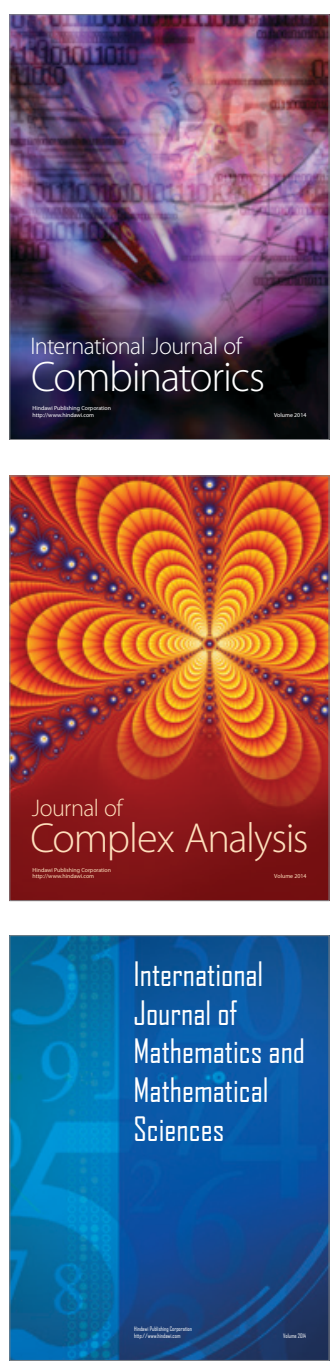
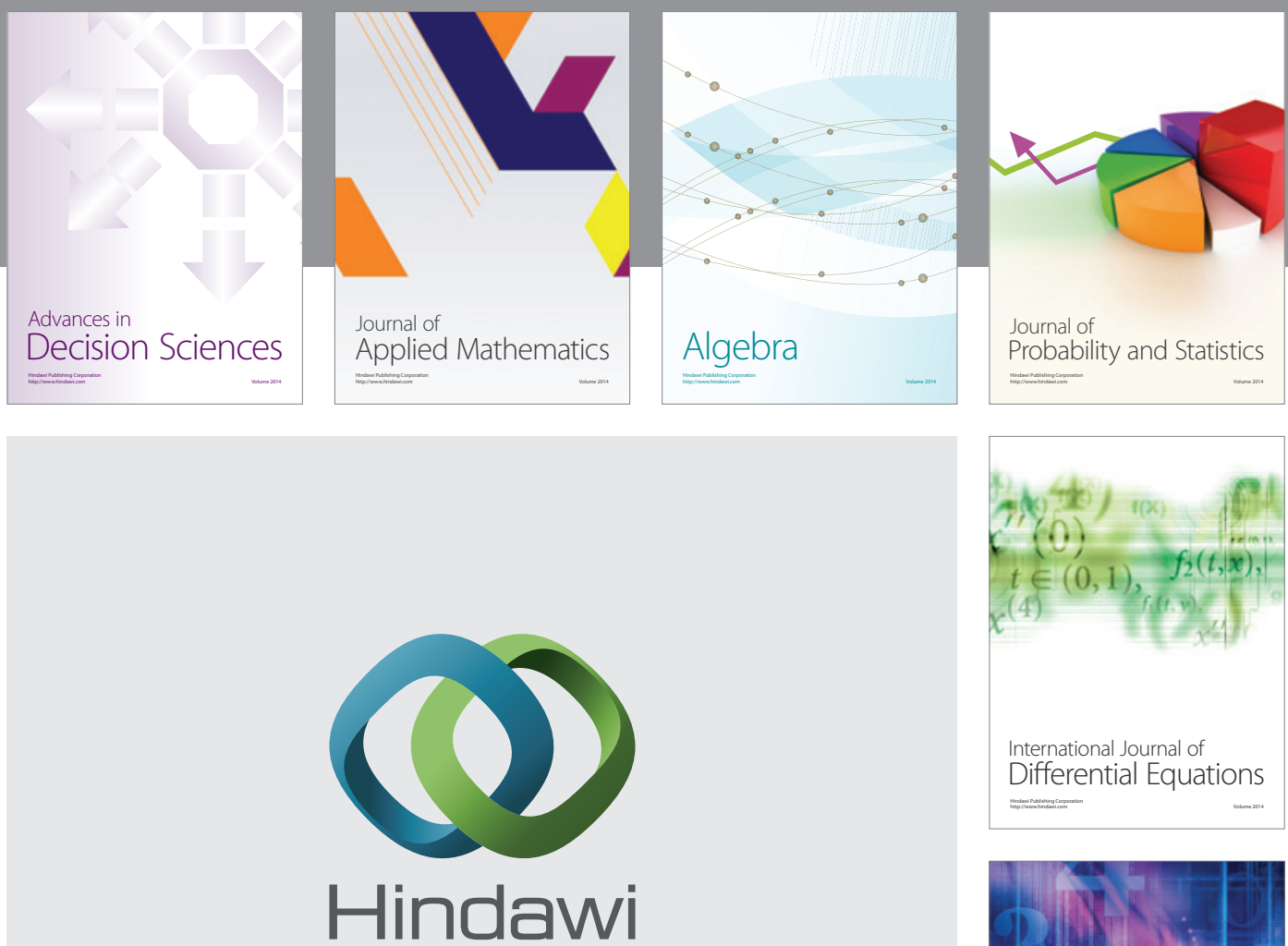

Submit your manuscripts at http://www.hindawi.com
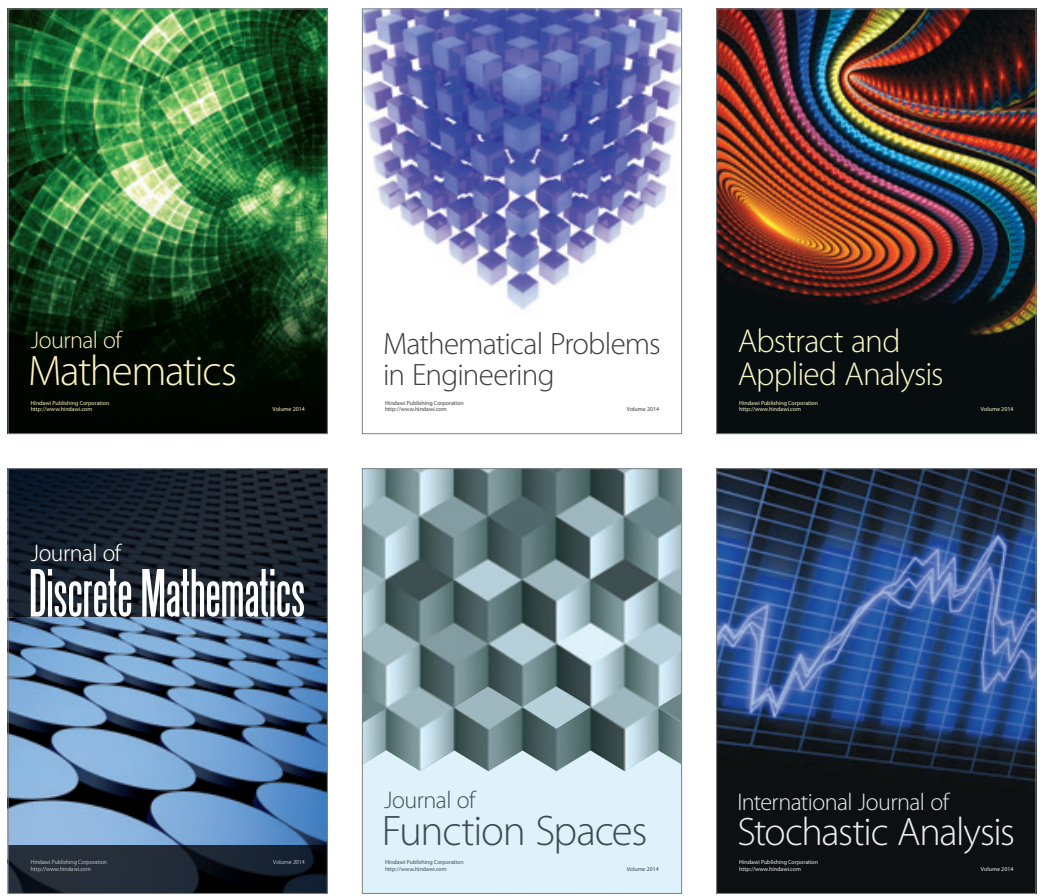

Journal of

Function Spaces

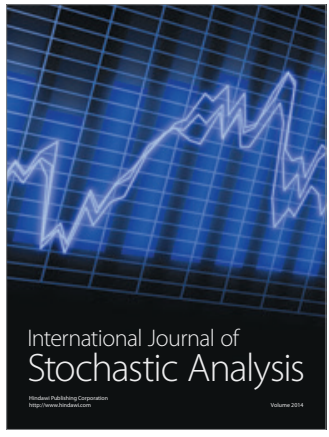

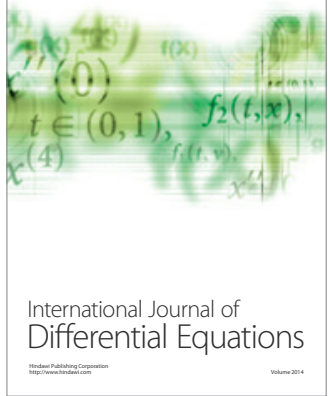
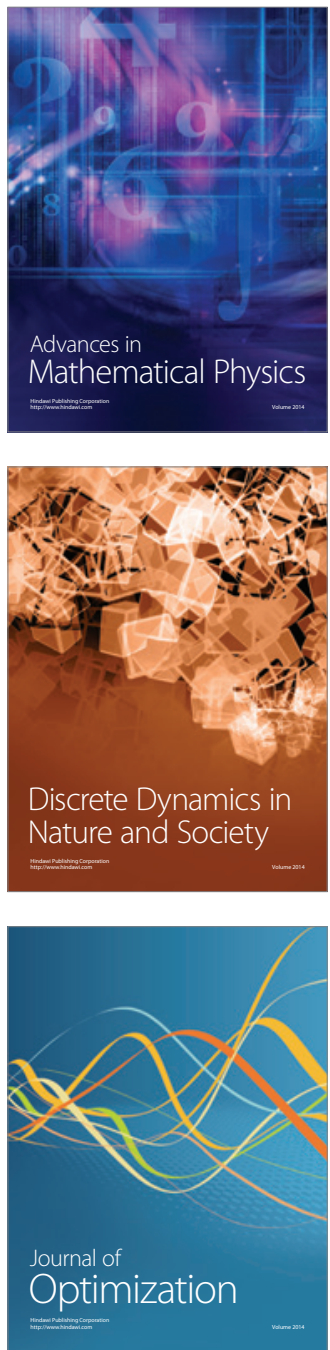